\title{
Recursos fisioterapêuticos no manejo da dor durante o trabalho de parto: uma revisão bibliográfica
}

\author{
Physiotherapeutic resources in pain management during labor: a literature review \\ Recursos fisioterapéuticos en el manejo del dolor durante el trabajo de parto: revisión de la
}

\section{Resumo}

O fisioterapeuta tem como atuação na obstetrícia auxilar e preparar a mulher para o trabalho de parto de parto. Sendo assim, o objetivo desse estudo foi identificar os recursos que auxiliam os fisioterapeutas no manejo da dor durante o trabalho de parto. Tratando-se de uma revisão bibliográfica com uma abordagem qualitativa, sendo inclusos estudos publicados entre os anos de 2016 e 2021, nos idiomas português e inglês, nas bases de dados: PubMed, PEDro (Physiotherapy Evidence Database) e Google Acadêmico. Os artigos inclusos foram todos de cunho experimental, realizados em humanos, por fim sendo selecionados 13 artigos que se encaixavam nos critérios de inclusão. Os recursos encontrados na literatura para o manejo da dor durante o trabalho de parto foram: hidroterapia (banho quente ou de imersão) (23.1\%), acupressão (23.1\%), estimulação elétrica nervosa transcutânea (TENS) (23.1\%), massagem $(30,7 \%)$, termoterapia (frio e/ou calor) $(15,3 \%)$ e exercícios respiratórios $(7,7 \%)$. Concluiu-se que os recursos encontrados mostram ser eficazes para o controle da dor durante o trabalho de parto e o conhecimento sobre eles se faz necessário para melhor assistir as parturientes nesse processo.

Palavras-chave: Dor; Trabalho de parto; Fisioterapia.

\begin{abstract}
The physiotherapist works in auxiliary obstetrics and prepares the woman for labor and delivery. Therefore, the aim of this study was to identify the resources that help physical therapists in managing pain during labor. This is a literature review with a qualitative approach, including studies published between 2016 and 2021, in Portuguese and English, in the databases: PubMed, PEDro (Physiotherapy Evidence Database) and Academic Google. The articles included were all of an experimental nature, carried out in humans, and finally 13 articles that met the inclusion criteria were selected. Resources found in the literature for pain management during labor were: hydrotherapy (hot bath or immersion) (23.1\%), acupressure (23.1\%), transcutaneous electrical nerve stimulation (TENS) (23.1\%), massage $(30.7 \%)$, thermotherapy (cold and/or heat) $(15.3 \%)$ and breathing exercises $(7.7 \%)$. It was concluded that the resources found show to be effective for pain control during labor and knowledge about them is necessary to better assist the parturients in this process.
\end{abstract}

Keywords: Pain; Labor; Physiotherapy.

\section{Resumen}

El fisioterapeuta trabaja en obstetricia auxiliar y prepara a la mujer para el trabajo de parto y el parto. Por lo tanto, el objetivo de este estudio fue identificar los recursos que ayudan a los fisioterapeutas a controlar el dolor durante el trabajo de parto. Se trata de una revisión de la literatura con enfoque cualitativo, que incluye estudios publicados entre 2016 y 2021, en portugués e inglés, en las bases de datos: PubMed, PEDro (Physiotherapy Evidence Database) y Academic Google. Los artículos incluidos fueron todos de carácter experimental, realizados en humanos, y finalmente se seleccionaron 13 artículos que cumplieron con los criterios de inclusión. Los recursos encontrados en la literatura para el manejo del dolor durante el trabajo de parto fueron: hidroterapia (baño caliente o inmersión) $(23,1 \%)$, acupresión $(23,1 \%)$, estimulación nerviosa eléctrica transcutánea (TENS) $(23,1 \%)$, masaje (30,7\%), termoterapia (frío 
y / o calor) (15,3\%) y ejercicios respiratorios (7,7\%). Se concluyó que los recursos encontrados demuestran ser efectivos para el control del dolor durante el trabajo de parto y es necesario conocerlos para asistir mejor a las parturientas en este processo.

Palabras clave: Dolor; Trabajo de parto; Fisioterapia.

\section{Introdução}

Antes do processo de institucionalização do parto, era comum mulheres passarem pelo processo de parir em domicílio com auxílio de "parteiras" e de seus familiares (Brüggemann, et al., 2005). Contudo, a partir da mudança do parto em domicílio para o ambiente hospitalar, o médico toma o papel ativo, fazendo com que a mulher não seja mais protagonista da condução de seu parto, não tendo mais autonomia sobre as escolhas feitas sobre sua saúde e seu corpo Desta forma, de acordo com Firmino, et al. (2020), a falta de profissionais capacitados para atender esse público piora a visão e a experiência dessas parturientes sobre o parto, assim criando um cenário traumatizante de sofrimento e dor física.

A dor referida ao trabalho de parto é relativa de cada gestante, já que envolve fatores psicológicos, emocionais, culturais, biológicos e socioeconômicos relacionados a cada uma destas (Nilsen, et al., 2011). Outro fator a ser levado em consideração é a expectativa criada pela parturiente para esse momento, que muitas das vezes esperam um processo sem dor (Firmino, et al., 2020).

Segundo Melo, et al. (2020) diante da dor, o corpo da mulher tende a reagir ao estresse sobrecarregando certos sistemas, como o sistema linfático, endócrino e cardiorrespiratório, aumentando a pressão sanguínea, sudorese e frequência respiratória. Para amenizar essas mudanças são várias as técnicas utilizadas no momento de parturição, a maioria são de caráter invasivo e medicamentoso que em muitos casos são danosos para a saúde materno e perinatais (Mielke, et al., 2019; Bavaresco, et al., 2011).

A atuação fisioterapêutica em obstetrícia auxilia na preparação e conscientização da parturiente sobre o trabalho de parto, tendo como objetivo conduzir a gestante a ser protagonista de todo o processo, vivenciando-o de forma plena e ativa, tendo controle da situação e assim tornando um momento satisfatório e com menores riscos obstétricos (Freitas, et al., 2017).

Segundo o estudo de Rocha, et al. (2020) a mulher mantendo-se ativa e em posições verticais tem como consequência a diminuição do tempo de trabalho de parto, além da dor e traumas perineal. As técnicas e métodos encontrados na literatura que podem ser relacionadas a atuação fisioterapêutica, que estão pertinentes a manter a parturiente ativa e amenizar os desconfortos, são: deambulação, exercícios com a bola suíça e massagem, banho quente, posicionamento vertical ou quatro apoios, exercícios respiratórios e analgesia com estimulação elétrica nervosa transcutânea (TENS) (Silva, et al., 2011; Bavaresco, et al., 2011). A introdução dessas técnicas nesse processo tem comprovações benéficas no manejo da dor e ansiedade, tornando uma assistência humanizada (Mielke, et al., 2019).

Um dos papeis mais importantes dos profissionais envolvidos nessa área é de promover o bem-estar e a segurança da parturiente, e para que isto aconteça é necessário ter conhecimento das melhores técnicas e métodos a serem utilizados para controle da dor durante o trabalho de parto. Sendo assim este estudo tem como objetivo identificar os principais recursos não farmacológicos que auxiliam os fisioterapeutas no manejo da dor durante o trabalho de parto.

\section{Metodologia}

Trata-se de uma revisão bibliográfica, pois segundo Koche (2011) revisão bibliográfica é o tipo de estudo que através de publicações na literatura tenta explicar um problema, tendo uma abordagem qualitativa, que teve como questão norteadora: “quais os recursos podem ser utilizados pelos fisioterapeutas no trabalho de parto para auxiliar no controle da dor?". Tendo como critérios de inclusão: artigos originais indexados no período dos últimos 5 anos (2016-2021), sendo estes de cunho experimental, como ensaios clínicos, randomizados ou não, realizados em humanos. Não sendo incluso: estudos de revisão; 
estudos que foram publicados no período anterior a 2016; artigos publicados, mas não concluídos; resumos, estudos com amostra menor que 50 mulheres, estudos que de forma geral não se encaixam no tema da presente revisão.

O levantamento de artigos foi feito através das seguintes plataformas de base de dados: PEDro (Physiotherapy Evidence Database), LILACS (Literatura Latino-Americana e do Caribe em Ciências da Saúde) e Google Acadêmico. Os termos labor pain, labor pain relief, therapy complemntary, foram combinados conforme a necessidade de cada base de dados, assim sendo possível encontrar estudos que melhor encaixavam-se nos critérios pré-estabelecidos.

Diante disso foi feita a seleção dos artigos, primeiramente fazendo identificação pelo título, seguido pela triagem, fazendo parte a leitura dos resumos dos artigos e por fim lidos por completos e selecionando os que eram elegíveis, que se encaixavam nos critérios de inclusão, assim finalizando em uma amostra de 13 artigos.

\section{Resultados e Discussão}

Os artigos selecionados foram todos de cunho experimental datados entre o período de 2016 - 2021. Foram selecionados 13 artigos que se encaixavam nos critérios de inclusão. Em suma, os artigos selecionaram gestantes de risco habitual, com idade gestacional a partir de 37 semanas e maiores de 18 anos. Para avaliar o nível de dor fora usado a Escala Visual Analógica (EVA) de dor, além de ter sido usado questionários pós-intervenção.

Os recursos encontrados na literatura para o manejo da dor durante o trabalho de parto foram: hidroterapia (banho quente ou de imersão) (23.1\%), acupressão (23.1\%), estimulação elétrica nervosa transcutânea (TENS) (23.1\%), massagem (30,7\%), termoterapia (frio e/ou calor) (15,3\%) e exercícios respiratórios (7,7\%). No Quadro 1 mostra os artigos selecionados, detalhando-os sobre o objetivo do estudo, tipo de recurso e seus resultados.

Quadro 1. Artigos selecionados.

\begin{tabular}{|c|c|c|c|c|}
\hline Autor e ano & Objetivo & Amostra & Intervenção/recurso & Resultados \\
\hline $\begin{array}{c}\text { Kaçar \& Keser, } \\
2021\end{array}$ & $\begin{array}{l}\text { Comparar os efeitos } \\
\text { da massagem } \\
\text { mecânica (luva) e da } \\
\text { massagem quente } \\
\text { (compressa) sobre a } \\
\text { redução da dor do } \\
\text { parto e satisfação de } \\
\text { mulheres primíparas. }\end{array}$ & 210 parturientes. & $\begin{array}{l}\text { Massagem mecânica, realizada } \\
\text { com luva de massagem, e } \\
\text { massagem quente, realizada } \\
\text { com pacote de cereja aquecida } \\
\text { no micro-ondas. Com duração } \\
\text { de } 15 \text { minutos após dilatação } \\
\text { cervical de } 4-5 \mathrm{~cm} \text { e } 7-8 \mathrm{~cm} \text {. }\end{array}$ & $\begin{array}{l}\text { Teve significativa diferença } \\
\text { na escala analógica de dor } \\
\text { após intervenção de } \\
\text { massagem quente. E maior } \\
\text { satisfação no grupo de } \\
\text { intervenção do que no de } \\
\text { controle. }\end{array}$ \\
\hline $\begin{array}{c}\text { Gönenç \& } \\
\text { Terzioğlu, } 2020\end{array}$ & $\begin{array}{l}\text { Avaliar o efeito da } \\
\text { acupressão e } \\
\text { massagem, isolados e } \\
\text { combinados, no } \\
\text { controle da dor do } \\
\text { parto. }\end{array}$ & 120 parturientes & $\begin{array}{l}\text { Massagem pelo corpo, por } 30 \\
\text { minutos na fase de dilatação de } \\
3-4 \mathrm{~cm}, 6-7 \mathrm{~cm} \text { e } 8-9 \mathrm{~cm} ; \\
\text { Acupressão no ponto de } \\
\text { Sanyinjiao (SP6), nos mesmos } \\
\text { períodos de dilatação e mesmo } \\
\text { tempo de duração. De forma } \\
\text { isolada e combinada }\end{array}$ & $\begin{array}{c}\text { A intervenção de massagem } \\
\text { + acupressão obteve maior } \\
\text { diferença no score de dor, } \\
\text { seguido por massagem e } \\
\text { acupressão, de forma } \\
\text { isolada. De forma } \\
\text { combinada resultou em } \\
\text { maior satisfação das } \\
\text { mulheres. }\end{array}$ \\
\hline $\begin{array}{c}\text { Cavalcanti, et al., } \\
2019\end{array}$ & $\begin{array}{l}\text { Através do uso de } \\
\text { banho quente, } \\
\text { exercícios com bola } \\
\text { suíça, de forma } \\
\text { isolada e combinada, } \\
\text { avaliar o efeito desses } \\
\text { sob a percepção de } \\
\text { dor, ansiedade e } \\
\text { progres-são do } \\
\text { trabalho de parto. }\end{array}$ & 128 parturientes & $\begin{array}{l}\text { Banho quente a } 37^{\circ} \mathrm{C}, \\
\text { direcionado jatos de água para } \\
\text { região lombo-sacra por } 15 \\
\text { minutos; Exercícios perineais } \\
\text { com bola suíça, realizados com } \\
\text { a parturiente sentada sobre a } \\
\text { bola fazendo movimentos de } \\
\text { propulsão e rotação pélvica por } \\
30 \text { minutos. Feitos de forma } \\
\text { isolada e combinada, sendo essa } \\
\text { por } 30 \text { minutos. }\end{array}$ & $\begin{array}{c}\text { Não teve redução da } \\
\text { percepção da dor, porém } \\
\text { teve melhora do quadro de } \\
\text { ansiedade das parturientes } \\
\text { e, quando combinadas, teve } \\
\text { diminuição do tempo de } \\
\text { evolução do parto. }\end{array}$ \\
\hline
\end{tabular}




\begin{tabular}{|c|c|c|c|c|}
\hline Cappeli, 2018 & $\begin{array}{l}\text { Avaliar a eficácia da } \\
\text { estimulação elétrica } \\
\text { nervosa transcutânea } \\
\text { (TENS) aplicada para } \\
\text { alívio de dor durante } \\
\text { trabalho de parto. }\end{array}$ & 68 parturientes & $\begin{array}{l}\text { Estimulação elétrica nervosa } \\
\text { transcutânea (TENS), utilizando } \\
\text { um par de eletrodos } \\
\text { posicionados entre a } 10^{\mathrm{a}} \\
\text { vértebra torácica (T10) e a } 1^{\mathrm{a}} \\
\text { lombar (L1) e outro par de } \\
\text { eletrodos entre a } 2^{\mathrm{a}} \mathrm{e} \text { a } 4^{\mathrm{a}} \\
\text { vértebra sacral (S2-S4). Com } \\
\text { uma frequência de } 100 \mathrm{~Hz} \text { e } \\
\text { largura de pulso de } 250 \mu \mathrm{s}, \text { com } \\
\text { duracão de } 30 \text { minutos. }\end{array}$ & $\begin{array}{l}\text { Diminuiu a intensidade de } \\
\text { dor e desconforto na fase } \\
\text { ativa do trabalho de parto }\end{array}$ \\
\hline $\begin{array}{c}\text { Báez-Suárez, et al., } \\
2018\end{array}$ & $\begin{array}{c}\text { Analisar sobre o } \\
\text { alívio da dor durante } \\
\text { o trabalho de parto } \\
\text { através da } \\
\text { estimulação elétrica } \\
\text { cutânea e a melhor } \\
\text { dosagem. }\end{array}$ & 63 parturientes & $\begin{array}{c}\text { Estimulação elétrica nervosa } \\
\text { transcutânea (TENS). Eletrodos } \\
\text { posicionados entre as vértebras } \\
\text { T10 -L1 e S2-S4. Na } \\
\text { intervenção } 1 \text { foi usado a uma } \\
\text { frequência de } 100 \mathrm{~Hz} \text { e largura } \\
\text { de pulso de } 100 \mu \text { s. Na } \\
\text { intervenção } 2 \text { foi utilizada uma } \\
\text { frequência variante entre } 80- \\
100 \mathrm{~Hz} \text { e largura de pulso } 350 \\
\mu \mathrm{s} \text {. As duas intervenções } \\
\text { tiveram duração de } 30 \text { minutos. }\end{array}$ & $\begin{array}{l}\text { Mostrou-se eficaz para } \\
\text { alívio da dor, } \\
\text { principalmente com } \\
\text { modulação de frequência de } \\
\text { 80-100Hz e largura de pulso } \\
\text { 350 } \mu \text { s }\end{array}$ \\
\hline Gallo, et al., 2018 & $\begin{array}{l}\text { Analisar o efeito dos } \\
\text { métodos não } \\
\text { farmacológicos sobre } \\
\text { o trabalho de parto }\end{array}$ & 80 parturientes & $\begin{array}{c}\text { Primeiramente foi feito } \\
\text { exercícios na bola suíça durante } \\
\text { a fase de } 4 \text { a } 5 \mathrm{~cm} \text { de dilatação } \\
\text { cervical; segundamente foi feito } \\
\text { massagem lombo-sacra quando } \\
\text { estava em 5-6 de dilatação; e } \\
\text { terceiro banho quente quando a } \\
\text { dilatação era maior que } 7 \mathrm{~cm} \text {. } \\
\text { As intervenções tinham duração } \\
\text { de } 40 \text { minutos, cada uma, } \\
\text { podendo durar menos conforme } \\
\text { a evolução do parto. }\end{array}$ & $\begin{array}{l}\text { Diminuição da dor e } \\
\text { consequentemente } \\
\text { diminuição do uso de } \\
\text { métodos farmacológicos. }\end{array}$ \\
\hline Czech, et al., 2018 & $\begin{array}{l}\text { Comparar eficácia de } \\
\text { métodos } \\
\text { farmacológicos e não } \\
\text { farmacológicos e } \\
\text { encontrar um padrão } \\
\text { ouro para alívio da } \\
\text { dor do parto }\end{array}$ & 258 parturientes & $\begin{array}{l}\text { Anestesia peridural; imersão em } \\
\text { água e nascimento na água; gás } \\
\text { de óxido nitroso; TENS; } \\
\text { múltiplos (gás + imersão em } \\
\text { água ou gás + TENS). As } \\
\text { mulheres escolhiam a conduta } \\
\text { que queriam seguir, em casos } \\
\text { de maior nível de dor podia ser } \\
\text { escolhido mais de um recurso. }\end{array}$ & $\begin{array}{c}\text { O método definido como } \\
\text { padrão ouro para alívio da } \\
\text { dor foi a anestesia peridural } \\
\text { e o método mais aceito } \\
\text { pelas mulheres foi o banho } \\
\text { de imersão. }\end{array}$ \\
\hline Yuksel, et al., 2017 & $\begin{array}{l}\text { Avaliar a eficácia de } \\
\text { exercícios } \\
\text { respiratórios durante } \\
\text { trabalho de parto } \\
\text { sobre dor de } \\
\text { parâmetros de saúde } \\
\text { do neonato. }\end{array}$ & 250 parturientes & $\begin{array}{l}\text { Receberam uma sessão de } \\
\text { exercícios respiratórios durante } \\
\text { a primeira fase do parto. Já na } \\
\text { segunda fase do parto } \\
\text { receberam instruções para fazer } \\
\text { respiração abdominal por conta } \\
\text { própria, "empurrando" o bebê } \\
\text { após uma inspiração profunda, } \\
\text { fazendo apneia ou expiração } \\
\text { lenta. }\end{array}$ & $\begin{array}{c}\text { Obteve controle relevante } \\
\text { da dor durante o segundo } \\
\text { estágio do trabalho de parto } \\
\text { e redução da duração. Sem } \\
\text { significativas diferença } \\
\text { entre os grupos em relação } \\
\text { a parâmetros de saúde dos } \\
\text { neonatos. }\end{array}$ \\
\hline $\begin{array}{l}\text { Mafetoni \& Shimo, } \\
2016\end{array}$ & $\begin{array}{l}\text { Analisar o efeito da } \\
\text { acupressão no ponto } \\
\text { B6 sobre a dor na fase } \\
\text { ativa do trabalho de } \\
\text { parto. }\end{array}$ & 156 parturientes & $\begin{array}{l}\text { No grupo de intervenção } \\
\text { receberam acupressão (pressão } \\
\text { profundo seguida de retirada } \\
\text { brusca com dedo polegar); o } \\
\text { grupo placebo recebeu uma } \\
\text { pressão superficial, os dois } \\
\text { grupos fizeram pressão no } \\
\text { ponto Sanyinjiao (BP6), por } 20 \\
\text { minutos; e no grupo de controle } \\
\text { tiveram o tratamento comum da } \\
\text { unidade obstétrica. }\end{array}$ & $\begin{array}{l}\text { Redução da dor no grupo } \\
\text { que recebeu a intervenção, } \\
\text { porém com pouco redução } \\
\text { no nível de dor, sendo } \\
\text { eficaz até } 8 \mathrm{~cm} \text { de dilatação. }\end{array}$ \\
\hline
\end{tabular}




\begin{tabular}{|c|c|c|c|c|}
\hline Ozgoli, et al, 2016 & $\begin{array}{l}\text { Comparar efeito da } \\
\text { acupressão em } \\
\text { diferentes pontos (LI4 } \\
\text { e BL32) sobre a dor } \\
\text { da fase ativa do } \\
\text { trabalho de parto. }\end{array}$ & 105 parturientes & $\begin{array}{l}\text { O grupo de BL32 recebeu uma } \\
\text { pressão na região sacral; o } \\
\text { grupo de LI4 recebeu uma } \\
\text { pressão em região entre o } 1^{\circ} \text { e } \\
2^{\circ} \text { dedo da mão. A intervenção } \\
\text { era aplicada apenas durante as } \\
\text { contrações, durando por } 6 \\
\text { contrações. No grupo de } \\
\text { controle receberam cuidados } \\
\text { rotineiros da obstetrícia. }\end{array}$ & $\begin{array}{l}\text { Mostrou que os dois pontos } \\
\text { de acupressão tiveram } \\
\text { efeitos positivos para o } \\
\text { alívio da dor nas primeiras } \\
\text { fases do parto se comparado } \\
\text { com o grupo de controle. } \\
\text { Com pouca diferença entre } \\
\text { o grupo de BL32 e LI4, } \\
\text { sendo o grupo BL32 } \\
\text { ligeiramente mais eficaz no } \\
\text { controle da dor. . } \\
\end{array}$ \\
\hline $\begin{array}{c}\text { Ahmad-Shirvani \& } \\
\text { Ganji, } 2016\end{array}$ & $\begin{array}{l}\text { Comparar efeito do } \\
\text { calor e frio, separados } \\
\text { e intercalados, sobre a } \\
\text { dor do parto }\end{array}$ & 96 parturientes & $\begin{array}{l}\text { No grupo de calor foi utilizado } \\
\text { compressa de água morna com } \\
\text { temperatura de } 38-40^{\circ} \mathrm{C} \text { em } \\
\text { região de abdômen e lombar, } \\
\text { tendo a duração e repetição } \\
\text { relativas as contrações; no } \\
\text { grupo de frio foi usado uma } \\
\text { bolsa de gelo, nas mesmas } \\
\text { regiões, durante } 10 \text { minutos, } \\
\text { repetindo a cada } 30 \text { minutos } \\
\text { durante a fase ativa do parto, na } \\
\text { segunda fase do parto foi } \\
\text { colocada a bolsa sob o períneo } \\
\text { por } 5 \text { minutos, repetindo a cada } \\
15 \text { minutos. No grupo de } \\
\text { alternância foi usado primeiro } \\
\text { por } 30 \text { minutos compressa } \\
\text { quente e depois por } 10 \text { minutos } \\
\text { de fria, repetindo a cada } 30 \\
\text { minutos. }\end{array}$ & $\begin{array}{l}\text { Não teve significativa } \\
\text { diferença entre as } \\
\text { intervenções de calor e frio, } \\
\text { as duas mostraram-se } \\
\text { convenientes para controle } \\
\text { da dor do parto. O grupo de } \\
\text { calor e de alternância } \\
\text { tiveram maior índice de } \\
\text { satisfação. }\end{array}$ \\
\hline $\begin{array}{l}\text { Lamadah \& } \\
\text { Nomani, } 2016\end{array}$ & $\begin{array}{l}\text { Avaliar o efeito da } \\
\text { massagem de } \\
\text { aromaterapia com } \\
\text { óleo de lavanda sobre } \\
\text { o nível de dor e } \\
\text { ansiedade do parto. }\end{array}$ & 60 parturientes & $\begin{array}{l}\text { No grupo de intervenção foi } \\
\text { feito massagem com } 2 \text { gotas de } \\
\text { óleo de lavanda dissolvido em } \\
50 \text { cc de óleo de amêndoa, no } \\
\text { grupo de controle foi feita } \\
\text { massagem sem óleo. Foi feita a } \\
\text { massagem nos dois grupos em } \\
\text { decúbito lateral por } 20 \text { minutos, } \\
\text { durante a fase ativa (5-7 cm de } \\
\text { dilatação) e na fase de transição } \\
\text { (8-10 cm de dilatação). }\end{array}$ & $\begin{array}{l}\text { O grupo de intervenção } \\
\text { obteve significativa redução } \\
\text { no score de dor comprado } \\
\text { ao grupo de controle, além } \\
\text { da redução do nível de } \\
\text { ansiedade e tempo de } \\
\text { trabalho de parto durante o } \\
\text { segundo estágio. }\end{array}$ \\
\hline $\begin{array}{l}\text { Akbarzadeh, et al., } \\
2016\end{array}$ & $\begin{array}{l}\text { Avaliar o efeito de } \\
\text { alívio da dor com } \\
\text { compressa quente em } \\
\text { dois estágios do } \\
\text { trabalho de parto. }\end{array}$ & 150 parturientes & Compressa quente & $\begin{array}{l}\text { Teve diminuição do tempo } \\
\text { da segunda fase do trabalho } \\
\text { de parto no grupo de } \\
\text { intervenção. }\end{array}$ \\
\hline
\end{tabular}

Fonte: Autores (2021).

A partir dos estudos expostos no Quadro 1 é possível notar que, dentre os recursos utilizados, os mais relatados na literatura são massagem e banho quente (aspersão ou imersão), sendo o banho quente muita bem aceito pelas parturientes e tendo um alto nível de satisfação e isso pode ser justificado, segundo Silva, et al. (2009), pelo fato de que através da água quente aconteça vasodilatação, liberação de substâncias como as catecolaminas e endorfina, possibilitando melhora do fluxo sanguíneo, relaxamento da musculatura e consequentemente melhora do estresse. Assim como no banho quente a massagem promove relaxamento, pois funciona através do toque e da manipulação dos tecidos que transmitem uma estimulação sensorial, aumentando a oxigenação dos tecidos e reduzindo o estresse (Araújo, et al., 2018).

Em um estudo de Hanum, et al. (2017) mostrou que mais 80\% das mulheres entrevistadas que utilizaram banho morno durante o trabalho de parto tiveram uma experiência satisfatória, além de mostrar que o maior índice de satisfação foi quando os métodos eram aplicados por outras pessoas, acompanhante ou profissional, mostrando a necessidade de uma equipe 
preparada.

No estudo de Barbieri, et al., (2013) concluiu que o uso de terapias complementares, como banho quente e exercícios com bola suíça, são mais eficazes para diminuição da dor quando usados de forma combinada, corroborando com os estudos de Gönenç e Terzioğlu (2020) e de Gallo, et al. (2018) que usaram recursos combinados e tiveram maior diferença no score de dor quando usado os recursos de forma conjunta ou alternada.

Os pontos de acupuntura e acupressão usualmente utilizados durante o trabalho de parto são Hegu (LI4) e Sanyinjiao (SP6). Sendo o ponto Sanyinjiao (SP6) para diminuição do tempo de parto, aumentando as contrações uterinas e o ponto Hegu (LI4) para diminuição da percepção da dor (Nwanodi, 2016). Essa afirmativa pode justificar o resultado de Gönenç e Terzioğlu (2020), que obteve pouca diferença para diminuição da dor quando usado apenas acupressão no ponto Sanyinjiao (SP6). Com os estudos exposto nesta revisão observa-se que a acupressão tem pouca eficácia sob o controle da dor do parto.

Outro recurso descrito pela literatura foi a estimulação elétrica transcutânea (TENS), no entanto ainda causa muitas controvérsias na literatura, ainda mais quando usada durante o trabalho de parto, muitos estudos questionam a eficácia dos efeitos propostos, como no estudo de Angelo, et al. (2016) que foi discutido o baixo nível metodológico dos artigos que usaram TENS como método de alívio da dor durante o trabalho de parto, tendo pouca evidência dos efeitos. Todavia, em estudos mais atuais como de Cappeli (2018) e Báez-Suárez, et al. (2018) expostos na presente revisão, com uma amostra mais consistente, mostra a eficácia do método, não apenas para alívio da dor, mas também para retardo de intervenções farmacológicas. Em um estudo randomizado de Santana, et al. (2016), aplicou-se TENS por 30 minutos em 46 parturientes durante a fase de dilatação e obteve resultados satisfatórios para alívio da dor, não tendo efeitos deletérios para saúde materno ou do neonatal e ainda incluiu o relato das mulheres sobre o diferencial positivo da assistência prestada durante esse processo.

Sendo assim, visando a diminuição das intervenções invasivas e medicamentosas, o mais indicado é fazer o manejo da dor através de recursos não invasivos, assim melhorando a evolução do parto e evitando as complicações obstétricas. Desta forma, deve-se os profissionais, tanto fisioterapeutas como outros envolvidos nessa área, estarem conscientes das possíveis intervenções a serem feitas para que isso ocorra.

Neste estudo pode ser identificadas algumas limitações inerentes a metodologia, como a restrição nas bases de dados e nos descritores.

\section{Conclusão}

Nesta pesquisa é possível notar que a maioria dos estudos que aplicaram recursos não farmacológicos tiveram como resultado a diminuição da sensação dolorosa e o tempo de progressão do parto. Os recursos mais bem aceitos pelas mulheres, que causam maior conforto e segurança, são os mais eficazes para o manejo da dor, mesmo esses não fazendo significativas mudanças no score de dor. Os que mais se destacam são os recursos de massagem, banho quente e TENS, sucessivamente. Os estudos encontrados não se relacionaram diretamente a atuação do fisioterapeuta, todavia, esses recursos podem ser plenamente usados pelos fisioterapeutas para assistir as parturientes durante o trabalho de parto.

Dessa forma, recomenda-se incentivar a elaboração de outros trabalhos científicos sobre o assunto, com intuito de enriquecer a comunidade acadêmica com conhecimento acerca do tema, e ampliar o número de pesquisas a respeito da fisioterapia obstétrica no manejo da dor durante o trabalho de parto.

\section{Referências}

Ahmad-Shirvani, M. \& Ganji, J. (2016). Comparison of separate and intermittent heat and cold therapy in labour pain management. Nurs Pract Today, 3(4), 179-186. https://npt.tums.ac.ir/index.php/npt/article/view/223

Akbarzadeh, M., et al. (2018). The Effect of Two-Staged Warm Compress on the Pain Duration of First and Second Labor Stages and Apgar Score in Prim Gravida Women: a Randomized Clinical Trial. J Car Sci., 7 (1), 21-6. 10.15171 / jcs.2018.004 
Angelo, P. H. M., et al. (2016). Recursos não farmacológicos: atuação da fisioterapia no trabalho de parto, uma revisão sistemática. Fisioterapia Brasil, 17(3): 285-292. https://doi.org/10.33233/fb.v17i3.489

Araújo, A. S. C., et al. (2018). Métodos não farmacológicos no parto domiciliar. Rev. Enferm. UFPE online, 12(4), 1091-6. https://doi.org/10.5205/1981-8963v12i4a230120p1091-1096-2018

Báez-Suárez, A., et al. (2018). Evaluation of different doses of transcutaneous nerve stimulation for pain relief during labour: a randomized controlled trial. Trials; 19, 652. https://doi.org/10.1186/s13063-018-3036-2

Barbieri, M., et al. (2013). Banho quente de aspersão, exercícios perineais com bola suíça e dor no trabalho de parto. Acta Paul Enferm, 26(5), 478-84. https://doi.org/10.1590/S0103-21002013000500012

Bavaresco, G. Z., et al. (2011). O fisioterapeuta como profissional de suporte à parturiente. Ciênc. saúde coletiva, 16(7), 3259-3266. https://doi.org/10.1590/S1413-81232011000800025

Brüggemann, O. M., et al. (2005). Evidências sobre o suporte durante o trabalho de parto/parto: uma revisão da literatura. Cad. Saúde Pública, 21(5), 13161327. http://dx.doi.org/10.1590/S0102-311X2005000500003.

Cappeli, A. J. (2018). Estudo randomizado do uso da Estimulação Elétrica Nervosa Transcutânea (TENS) no alívio da dor no trabalho de parto. Dissertação (Mestrado em Ginecologia, Obstetrícia e Mastologia) - Faculdade de Medicina, Universidade Estadual Paulista "Júlio de Mesquita Filho". Botucatu, 46 p. https://repositorio.unesp.br/bitstream/handle/11449/153472/cappeli_aj_me_bot.pdf;jsessionid=5079C0CB3F14BAD7E83DC8247967E750?sequence=3

Cavalcanti, A. C. V., et al. (2019). Terapias complementares no trabalho de parto: ensaio clínico randomizado. Rev. Gaúcha Enferm., 40 , e20190026. https://doi.org/10.1590/1983-1447.2019.20190026

Czech, I., et al. (2018). Pharmacological and Non-Pharmacological Methods of Labour Pain Relief-Establishment of Effectiveness and Comparison. Int. J. Environ. Res. Public Health, 15, 2792, 1-11. 10.3390 / ijerph15122792

Freitas, A. S., et al. (2017). Atuação da Fisioterapia no parto humanizado. Dê Ciência em Foco, 1(1), 18-29. http://revistas.uninorteac. com.br/index.php/DeCienciaemFoco0/article/view/15

Firmino, K. C., et al. (2020). Percepção da mulher frente à dor do parto. Revista Ciência Plural, 6(1), 87-101. https://doi.org/10.21680/24467286.2020v6n1ID18387

Gallo, R. B. S., et al. (2018). Sequential application of non-pharmacological interventions reduces the severity of labour pain, delays use of pharmacological analgesia, and improves some obstetric outcomes: a randomised trial. J Physiother, 390, 8. 10.1016 / j.jphys.2017.11.014

Gönenç, I. M. \& Terzioğlu, F. (2020). Effects of Massage and Acupressure on Relieving Labor Pain, Reducing Labor Time, and Increasing Delivery Satisfaction. The Journal of Nursing Research, 28(1), 1-9. 10.1097/jnr.0000000000000344

Hanum, S. P., et al. (2017). Estratégias não farmacológicas para o alívio da dor no trabalho de parto: efetividade sob a ótica da parturiente. Rev enferm UFPE online., v. 11(8), 3303-9. https://doi.org/10.5205/1981-8963-v11i8a110197p3303-3309-2017

Kaçar, N. \& Keser, N. O. (2021). Comparison of the effect of mechanical massage and warm mechanical massage application on perceived labor pain and childbirth experience: A randomized clinical trial. European Journal of Midwifery, 5(2), 1-10. 10.18332 / ejm / 132883

Koche, J. C. (2011). Fundamentos de metodologia cientifica. Vozes. http://www.adm.ufrpe.br/sites/ww4.deinfo.ufrpe.br/files/Fundame ntos_de_Metodologia_Cienti\%CC\%81fica.pdf

Lamadah, S. M. \& Nomani, I. (2016). The Effect of Aromatherapy Massage Using Lavender Oil on the Level of Pain and Anxiety During Labour Among Primigravida Women. American Journal of Nursing Science. 5(2), 37-44. 10.11648 / j.ajns.20160502.11

Mafetoni, R. R. \& Shimo, A. K. K. (2016). The effects of acupressure on labor pains during child birth: randomized clinical trial. Rev. Latino-Am. Enfermagem. 24:e2738. 10.1590/1518-8345.0739.2738

Melo, P. S. et al. (2020). Parâmetros maternos e perinatais após intervenções não farmacológicas: um ensaio clínico randomizado controlado. Acta paul. enferm., v. 33, eAPE20190136. https://doi.org/10.37689/acta-ape/2020ao0136

Motta, S. A. M. F., et al. (2016). Implementação da humanização da assistência ao parto natural. Rev. Enferm. UFPE online., 10(2), 593-9. 10.5205/reuol.8557-74661-1-SM1002201628

Nilsen, E., et al. (2011). Dor e comportamento de mulheres durante o trabalho de parto e parto em diferentes posições. Rev. esc. enferm. USP, 45(3), 557-565. https://doi.org/10.1590/S0080-62342011000300002

Nwanodi, O. B. (2016). Labor Pain Treated with Acupuncture or Acupressure. Chinese Medicine. Salinas, 7, 133-152. 10.4236 / cm.2016.74014

Ozgoli, G., et al. (2016). Effect of LI4 and BL32 acupressure on labor pain and delivery outcome in the first stage of labor in primiparous women: A randomized controlled trial. Complementary Therapies in Medicine, 29, 175-180. 10.1016 / j.ctim.2016.10.009

Rocha, B. D. et al. (2020). Posições verticalizadas no parto e a prevenção de lacerações perineais: revisão sistemática e metanálises. Rev Esc Enferm USP. 54, :e03610, 2020. https://doi.org/10.1590/S1980-220X2018027503610

Santana, L. S., et al. (2016). Transcutaneous electrical nerve stimulation (TENS) reduces pain and postpones the need for pharmacological analgesia during labour: a randomised trial. Journal of Physiotherapy, 62, 29-34. https://doi.org/10.1016/j.jphys.2015.11.002 
Research, Society and Development, v. 10, n. 12, e486101220610, 2021

(CC BY 4.0) | ISSN 2525-3409 | DOI: http://dx.doi.org/10.33448/rsd-v10i12.20610

Silva, F. M. B., et al. (2009). A randomised controlled trial evaluating the effect of immersion bath on labour pain. Midwifery, 25(3), 286-94. 10.1016/ j.midw.2007.04.006

Silva, L. M., et al. (2011). Uso da bola suíça no trabalho de parto. Acta paul. enferm., 24(5), 656-662. https://doi.org/10.1590/S0103-21002011000500010

Yuksel, H., et al. (2017). Effectiveness of breathing exercises during the second stage of labor on labor pain and duration: a randomized controlled trial. Journal of Integrative Medicine, 15(6), 456-461. 10.1016 / S2095-4964 (17) 60368-6 